\title{
Politicians-based Deep Learning Models for Detecting News, Authors and Media Political Ideology
}

\author{
Khudran M. Alzhrani \\ Department of Information Systems \\ Al-Qunfudhah Computing College, Umm Al-Qura University \\ Al-Qunfudhah, Mecca, Saudi Arabia
}

\begin{abstract}
Non-partisanship is one of the qualities that contribute to journalistic objectivity. Factual reporting alone cannot combat political polarization in the news media. News framing, agenda settings, and priming are influence mechanisms that lead to political polarization, but they are hard to identify. This paper attempts to automate the detection of two political science concepts in news coverage: politician personalization and political ideology. Politicians' news coverage personalization is a concept that encompasses one more of the influence mechanisms. Political ideologies are often associated with controversial topics such as abortion and health insurance. However, the paper prove that politicians' personalization is related to the political ideology of the news articles. Constructing deep neural network models based on politicians' personalization improved the performance of political ideology detection models. Also, deep networks models could predict news articles' politician personalization with a high F1 score. Despite being trained on less data, personalizedbased deep networks proved to be more capable of capturing the ideology of news articles than other non-personalized models. The dataset consists of two politician personalization labels, namely Obama and Trump, and two political ideology labels, Democrat and Republican. The results showed that politicians' personalization and political polarization exist in news articles, authors, and media sources.
\end{abstract}

Keywords-Deep neural networks; text classification; political ideology; politician personalization

\section{INTRODUCTION}

This paper examines the relationship between two political science concepts that usually addressed separately, news articles' political ideology and personalization. Political ideology of an article can be predicted based on its content, publisher ideology, or authors leanings. Our main hypothesis is that by constructing intelligent models trained on politician centered articles will improve their performance in detecting articles' political ideologies. Personalization attributes such as politician visibility, personal traits, and reoccurring topics form a pattern that statistical models learn to distinguish personalized articles. articles' tags are used in building the dataset, which indicate that the politician is directly related to the topic covered in the article. In the Presidential Dataset (Section III), Obama was mentioned in $23.6 \%$ of the articles that were tagged with Trump. Despite that, our models were able to detect articles personalization and the ideology of personalized articles effectively. Nonetheless, introducing intelligent models to detect articles' ideology based on their personalization proved to advance their performance.
One would question the motivation of paper by pointing out that the readers could identify politicians mentioned in the article and be aware of the article's writer or publisher's political alignment at the same time. However, not all readers are attentive [1], and political leanings of news sources or writers are not always known. Also, detailed articles might have few mentions of a politician and not be personalized. Some readers do not go beyond initial information exposure of shared articles, or cropped news on a website and news feeds [2]. Also, websites' structure differs from one another, meaning not all news websites use web tags. Some websites' main page displays articles in their entirety; others show the articles' title and maybe a snippet of its first paragraph. News agencies share links to the news articles on social media networks. Readers might arrive at conclusions solely based on the articles' headlines or posts on character-limited social networks. Automated personalization detection increases the amount of information available to readers, even without reading the article. The pre-trained models can easily detect who is personalized in this article and notify the reader. Models can be trained on multi-labeled data to detect more than one politician's personalization.

The research's interdisciplinary nature contributes towards bringing artificial intelligence to political science, journalism, and communication. The proposed concept has practical applications for regular readers, news outlets, social networking sites, and politicians. The intelligent models will provide awareness to the reader about personalized articles' ideologies. Labeling the article based on its political ideology helps the reader identify which standpoint the article takes. Hence, the reader is encouraged to seek the other viewpoints to determine which one is more convincing to align themselves to it or take a specific position. For instance, if most of the articles exhibited to a reader were from one political side, the reader's awareness of being in a bubble would increase.

News Recommender Systems (NRS) aims to customize the news articles displayed to the readers based on criteria or techniques that are believed to capture the readers' interest. Researchers have proposed several expert systems to recommend the news from the web [3], [4], news agencies' tweets [5], and heterogeneous data tailored for journalists [6]. Moreover, social networks integrate news recommendation systems into their platform, such as Facebook news feed, to suggest news articles based on users' behavior, etc. [7]. Due to deep neural networks' ability to overcome some of the 
limitations in the traditional techniques, the incorporation of deep neural networks into news recommendation systems have been more frequent in recent years [8], [9], [10]. Regardless of the adopted methods to build the news recommendation systems, researchers pointed out that such systems increase pro-attitudinal selective exposure of political news [11], [12], [13]. Articles' personalization and ideology detection models can help the news recommendation systems mitigate proattitudinal selective exposure.

Deep transfer learning for texts enables knowledge sharing between a source domain with a sufficient amount of labeled data to target domains that suffer from labeled data insufficiency. The transfer could be achieved by different means, e.g., pre-trained word embedding [14], instance-based [15], and adversarial-based [16]. Because the Presidential Datasets are reconstructed to target articles, authors, and sources, transferring the DNN to multiple domains is possible. Personalization of politicians is transferable to other personalization types as CEOs' news media coverage [17]. Researchers pointed out that the media treats CEOs as brands and that preserving the CEO's image improves products and firms' value [18]. Personality prediction [19] and framing and agenda-setting detection [20] are other examples of transfer learning destinations. Other domains that are suitable for transfer learning from ideology detection models are emotion detection [21], top-specific opinion classification [22], and news bias identification [23].

In this work, a new approach is proposed to improve the performance of news political ideology detection models by building models with feature space extracted from politicians' personalized articles. Politicians' personalized-based models for political ideology detection were able to achieve higher or match the performance of other non-personalized political detection models, which trained on much more data. In addition, The experiments proved that it is possible to detect articles politicians' personalization automatically. The detection models were evaluated against news articles, authors, and media sources to examine the relations between the two political concepts. Finally, a statistical analysis of the presidential dataset from the political personalization perspective is provided.

The reminaing of this paper is structured as follows: Section II briefly reviews news personalization literature and describes how this paper's contribution can fit in this research field. We analyze the statistics related to articles' personalization in the Presidential data and its relation to political ideology in Section III. Description of research models and experimental setup provided in Section IV. Section V report and discuss results of articles' personalization and ideology detection. Finally, the paper is concluded in Section VI.

\section{RELATED WORK}

The related work of the automated texts' ideology detection is extensively reviewed in [24]; therefore, it will not be covered in this paper because. In this section, the literature of political personalization in the news media is reviewed.

Personalization in the literature comes in many shapes and forms. Personalization could mean delivering services [25], advertisements [26], or educational content [27] that best suited for a targeted individual. In law, personalization is substituting a uniform law for one tailored to an individual's preference, characteristics, or circumstances [28]. Other fields have several definitions for personalization as in marketing [29] and ecommerce [30], leading to confusion or ambiguity. Similarly, scholars in political science and communication view personalization from different perspectives. Researchers have examined the existence of personalization in various types of political institutions [31]. Others focused on studying personalization impact on the leaders' [32] or the public [33] political behavior. Numerous studies investigated the role of personalization in political campaigns and elections [34], [33]. Some of the political scientists debated that the rise of personalization is beneficial for modern democracies - the following are some of the observations that support this claim. Leaders will advocate the party's message and increase citizens' political engagement through direct online communication [35]. Voters' attachment to parties weaken due to increased political personalization; hence, voters would be more willing to vote for a different party in subsequent elections [36]. On the other hand, some will argue that personalization brings more harm than good to democracy [37]. For example, loosen the ties between voters and parties might move voters' and political parties' attention from local to national elections [36]. The literature on political personalization is extensive and diverse. This paper is closely related to papers that address presidential personalization in the online news media.

Peoples' political disagreements on the personal level manifest itself by voting for candidates who align with their political ideology as in representative democracy. The political parties mostly nominate their preferred candidates according to their set of criteria. These candidates roles revolves on advocating and implementing the party's program once they get in the government. Therefore, to some degree, the voter, candidate, and the party all have the same political ideology; hence, voters tend to stick with one party to help advance its policies. Mughan [38] stated that in the sixties, the United Kingdom and United States voters' interest shifted from the political parties to an individual politician's personalities, especially those in the high government positions. The media attention moves from the party loyalty to the particular politician's personalities at the top to lead, namely presidentialization [38], [39], [40].

The use of presidentialization as terminology to describe the UK's prime ministries nominees' influence over the electoral process and the elected prime minister rise in power over their cabinet members is debated [41]. Other political scientists prefer the term prime ministerialisation [42] or personalization [43]. Furthermore, individuals attribute populism mounting to personalized politics, where political leaders with compelling personalities appeal to a broader range of voters [44], [45]. Although the degree of political personalization differs depending on multiple variables such as the number of competing parties [46], it is common to find political personalization across western democracies [47], [48], [49], [36]. In her comprehensive book [47], Bittner classified the divergent research of political personalization into five categories: leaders' selection, leaders' traits, leaders' evaluation, leaders' impact on electoral outcomes, and information sources. The media is considered one of the primary information sources available to voters, and hence the criticality of the media's depiction of a political candidate is critical. 
The waning of the power of monolithic politics gave rise to the media coverage of individual politicians. Aelst et al. [50] developed a model to organize the political news personalization studies into two dimensions logically. The first dimension compares political news coverage of individual politicians and political institutions, leading to a dimension known as individualization. The second dimension emphasizes the change in politicians' media coverage as a government official to a private citizen; hence, this dimension is labeled privatization.

The individualization stems from two decentralized branches, which examine any or all politicians' media visibility regardless of their position [50], [51]. It also includes centralized, which study's the media's visibility regarding different political leaders and their characteristics [52], [53], [46]. Relativity different take on the distinctions between centralized and decentralized media personalization articulated in [54] defined centralized personalization as the media visibility of an upward shift towards politicians at the top, On the other hand, researchers described decentralized personalization as a downward shift towards politicians in lower positions or parties. Privatization consists of two sub-dimensions [50], [55], personal characteristics and personal life. Personal characteristics refer to increased media coverage of a politician's traits rather than his political elements. Personal characteristics make people aware of a politician's positive or negative aspects as those who focus on those aspects aim at attaining certain political goals. On the other hand, personal life, which addresses media coverage, shifts to politician personal activities and interest. Research in politicians' traits is inconclusive since the dissimilarities between personal and political traits are clouded [56].

Others argue that personal disclosures in the media could be politicized [50]. Now that personalization concepts laid out in the previous paragraphs, one cannot place personalized media detection into these dimensions and subdimensions. We are not aware of any paper that researched the problem of news media personalization detection. However, one can link this work to some of those subdimensions. In personalized media detection, the deep network models learn politicians' personalities and political traits with other attributes to form a pattern in which one quickly identifies personalized articles. This research paper also touches on presidentialization or centralized personalization by experimenting with personalized articles regarding two US presidents,, namely President Donald and Trump and Barrack Obama. The two presidents have massive information attributed to them in the media and different other websites. The information is mostly published to sensitize the public about their aspects or public aspects crucial in politics. Despite that, personalized media detection should be distinguished from other previously known personalization dimensions. However, this distinguishing aspect does not eliminate the possibility of incorporating personalized media detection in different types of news personalization.

This paper is the first research paper that considered articles' political personalization as a dimension to detect ideologized text to the best of our knowledge. On top of that the ideology detection models' performance are evaluated in association with articles' authors and sources.
TABLE I. The Table Shows Size of Political Ideology and Political Personalization labels in the Presidential Dataset.

\begin{tabular}{cccccc} 
& & \multicolumn{2}{c}{ Ideology } & \multicolumn{2}{c}{ Personalization } \\
\cline { 3 - 6 } Set & \# Articles & Conservative & Liberal & Obama & Trump \\
\hline Train & 125051 & $35035(28 \%)$ & $90016(72 \%)$ & $72256(58 \%)$ & $52795(42 \%)$ \\
\hline Test & 53521 & $15017(28 \%)$ & $38504(72 \%)$ & $30896(58 \%)$ & $22625(42 \%)$ \\
\hline
\end{tabular}

TABle II. The TABle Shows the Total Number of Training AND TESTING ARTICLES FOR THE IDEOLOGY Classes IN TRUMP AND OBAMA DATA.

\begin{tabular}{lllll} 
& Trump Dataset & \multicolumn{3}{l}{ Obama Dataset } \\
\hline Class/Dataset & Train & Test & Train & Test \\
\hline Conservative & $22933(43.4 \%)$ & $9712(43 \%)$ & $12102(16.7 \%)$ & $5305(17.2 \%)$ \\
\hline Liberal & $29862(56.6 \%)$ & $12913(57 \%)$ & $60154(83.3 \%)$ & $25591(82.8)$ \\
\hline Total & 52795 & 22625 & 72256 & 30896 \\
\hline
\end{tabular}

\section{Personalization In the Presidential Dataset}

The articles in the Presidential dataset [57] were collected from multiple news sources aligned with extreme left and right on the political spectrum. A detailed description of the dataset and the methods used to collect the articles are provided in [24] and listed in Table I. The dataset is a collection of articles written about two U.S. presidents, Trump, and Obama. The two represent two different political ideologies and covered by all news agencies. The paper will focus on personalized articles in the dataset and its relation to the article's ideology, which were not explained in the previous publications. And examine how the dataset is balanced in terms of media attention to the politician and the ideology of media sources that published the articles.

Data imbalance is a common problem in text classification [58], and it hurts the performance of the detection models. As seen in Table (), the presidential dataset can be organized based on politician personalization, and political ideology. From personalization perspective Trump and Obama's articles represent about $42 \%$ and $58 \%$ of the entire corpus, respectively. On the other hand, $28 \%$ and $82 \%$ of the articles are labeled with Conservative and Liberal political ideologies, respectively. In comparison, personalized articles are more balanced than ideology articles. The proposed hypothesis assumes that constructing intelligent models trained on political personalized articles to detect political ideologies would improve the detection models performance. Knowing that personalized articles are more balanced than the ideology ones might contribute to this matter. However, one cannot be certain that the same could apply to the size of media coverage of other politicians. Politician serving times, position and other factors can generate more media attention.

Table II lists detailed statistics of personalized corpora. More than $43 \%$ of the articles written about Trump are conservative, and only $17 \%$ are labeled as conservative in the Obama corpus. As can be seen in Fig. 1, articles labeled as Liberal are more significant than Conservative articles in the Obama and Trump datasets. Moreover, the gap between the size of Conservative and Liberal articles is much more evident for Obama's articles. Therefore, one can assume that intelligent models would be able to detect the ideology of 
Table iII. The Table Displays News Sources, Ideology Alignments, and the Number of Articles in Trump and Obama Datasets.

\begin{tabular}{|c|c|c|c|}
\hline News Website & Ideology & Trump Dataset & Obama Dataset \\
\hline DailyWire [7.57\%] & Conservative & 11031 [14.62\%] (Tr:7797, Te:3234 ) & $2488[2.41 \%](\operatorname{Tr}: 1757, \mathrm{Te}: 731)$ \\
\hline ILoveMyFreedom [4.22\%] & Conservative & $6350[8.42 \%](\operatorname{Tr}: 4488$, Te:1862) & 1180 [1.14\%] (Tr:826, Te:354) \\
\hline DailyKos [68\%] & Liberal & $39822[52.8 \%](\operatorname{Tr}: 27808$, Te:12014 ) & 81691 [79.19\%] (Tr:57327, Te:24364) \\
\hline National Review [11.2\%] & Conservative & 9981 [13.23\%] (Tr:6929, Te:3052) & 9945 [9.64\%] (Tr:6880, Te:3065) \\
\hline TheBlaze $[0.62 \%]$ & Conservative & 614 [0.81\%] (Tr:432, Te:182) & $494[0.48 \%]$ (Tr:351, Te:143) \\
\hline WorldSocialist [3.92\%] & Liberal & 2953 [3.91\%] (Tr:2054, Te:899) & 4054 [3.93\%] (Tr:2827, Te:1227) \\
\hline NewsBusters [4.4\%] & Conservative & 4669 [6.19\%] (Tr:3287, Te:1382) & 3300 [3.2\%] (Tr:2288, Te:1012) \\
\hline Total & Combined & 75420 & 103152 \\
\hline
\end{tabular}

articles covering Trump better than Obama.

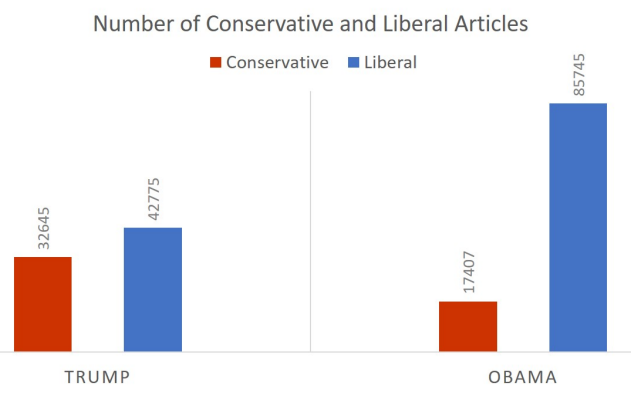

Fig. 1. Personalization V.S. Ideology. The Figure Shows the Number of Conservative and Liberal Articles Tagged with Trump and Obama.

The reason behind the difference in size among personalized and ideology articles can be understood better by exploring the number of articles generated by each media source. Table III presents all the news media sources' political ideology and the number of articles written about Trump and Obama. A large portion of the Presidential dataset comprises of articles from the DailyKos, $68 \%$ to be exact.

The difference in coverage becomes apparent in the horizontal bar chart in Fig. 2. DailyKos, a liberal media outlet, represents $52.8 \%$ and $79.19 \%$ of Trump and Obama datasets, respectively. The second and last liberal data source, WorldSocialist, is the only media source with consistent representation in the Presidential, Trump, and Obama datasets, which is, on average, $3.92 \%$. The remaining data sources are conservative and mostly have more written articles about Trump than Obama. One can conclude from this plot that all conservative sources, except for National Review Online, have more articles about Trump than Obama. On the other hand, Obama received more attention in the liberal media sources.

Parts of some articles in the Presidential Dataset are in Table IV. These articles' snippets provide the information needed to determine the authors' standpoints on controversial issues such as Obamacare, Iran's nuclear deal, and climate change. For instance, the far-left news source DailyKos article covering an event that took place in the Senate to repeal Obamacare pointed out the failure of the Republicans in the Senate to take health coverage from 16 million people. Another article reporting on the same event but from the conservative point of view stated that the modest Obamacare repeal offered by the Senate Republicans was 'killed' by the Democrats.

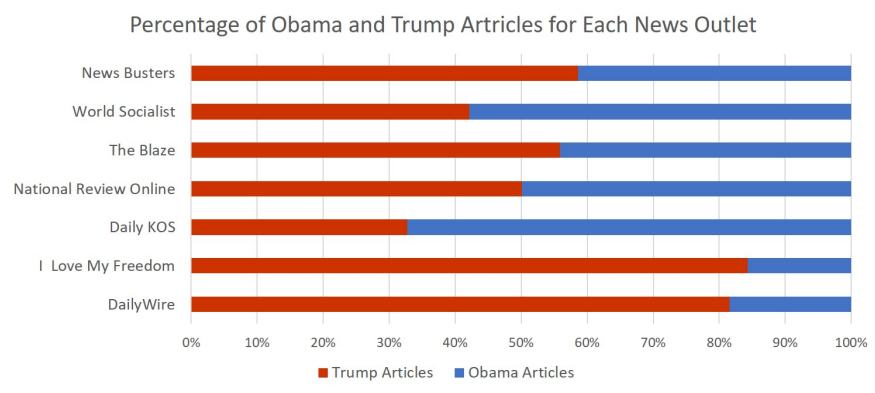

Fig. 2. Percentage of Personalized Articles in Media Sources. Display the Percentage of Articles Tagged with Trump and Obama in each Data Source.

Other examples are seen in the table and throughout the dataset. The experiment section will discuss the detection models trained on Trump and Obama datasets independently to detect their articles' political ideologies. Also, separate sets of the detection models will be trained on the presidential training set to identify Trump and Obama's personalized articles.

\section{RESEARCH Models AND EXPERIMENTAL SETUP}

Similar to [24], the following four deep neural network models are employed to detect news' articles politician personalization and political ideology.

1) FastText [59] models enable tasks to word representation fine tuning through averaging word vectors and updating embeddings in the training phase through back-propagation. The word embeddings are then fed to a fully-connected layer with Softmax activation to map articles representation to category labels. Articles word order is ignored during the construction of the text representation, which increase FastText classifiers speed and still achieve relatively good results.

2) Convolutional Neural Networks for the text classification (TextCNN) [60] use multiple convolution layers with different kernel sizes. In text classification, the convolution layers are effective in extracting features over multiple sliding windows from one dimensional inputs. The output of the convolution layers are max-pooled over the entire sequence to identify the most useful features and generate fixed-length vector. The features maps constructed from the max-pooling 


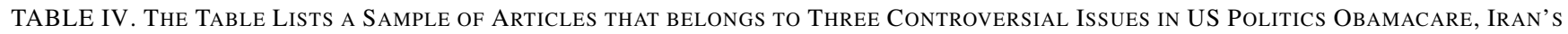
NuClear Deal, AND Climate CHANGE.

\begin{tabular}{|c|c|c|c|c|}
\hline Theme & Personalized/Ideology & Snippet From The Article & Date & Source \\
\hline \multirow[t]{4}{*}{ Obamacare } & Trump/Liberal & $\begin{array}{l}\text { Senate Republicans failed in their latest attempt to take health coverage from } \\
16 \text { million people with so-called "skinny repeal". }\end{array}$ & $07-28-2017$ & DailyKos \\
\hline & Trump/Conservative & $\begin{array}{l}\text { Around midnight Thursday, the Senate Republicans' attempt to pass even the most } \\
\text { modest of their Obamacare repeal efforts crashed and burned when "Maverick" } \\
\text { John McCain joined the so-called moderate Republicans Susan Collins (ME) and } \\
\text { Lisa Murkowski (AK) to vote with the } 48 \text { Democrats to kill the "skinny repeal" bill. }\end{array}$ & $07-28-2017$ & DailyWire \\
\hline & Obama/Conservative & $\begin{array}{l}\text { Democrats from Sen. Chuck Schumer (D-NY) to former President Barack Obama } \\
\text { announced this weekend that their first priority after the holidays will be to preserve } \\
\text { the Affordable Care Act which was declared unconstitutional (again) Friday by } \\
\text { a federal district court judge. }\end{array}$ & $12-16-2018$ & DailyWire \\
\hline & Obama/Liberal & $\begin{array}{l}\text { President Obama visited Texas last week. He had a chance to visit with Texas families } \\
\text { personally impacted by the health care law, Obamacare. }\end{array}$ & 04-18-2014 & DailyKos \\
\hline \multirow[t]{4}{*}{ Iran's Deal } & Trump/Liberal & $\begin{array}{l}\text { Donald Trump is looking for excuses to pull out of the Iran nuclear deal- } \\
\text { after all, it was negotiated under former President Obama, whose accomplishments } \\
\text { Trump is looking to wipe out one by one-even though his own top advisers like } \\
\text { Defense Secretary James Mattis and Joint Chiefs of Staff Chair Gen. Joseph Dunford } \\
\text { have said that the deal is working. }\end{array}$ & $10-10-2017$ & DailyKos \\
\hline & Trump/Conservative & $\begin{array}{l}\text { Donald Trump was exactly right when he called the Iran deal a "horrible" } \\
\text { and "disastrous" agreement. }\end{array}$ & $11-14-2016$ & NationalReview \\
\hline & Obama/Conservative & $\begin{array}{l}\text { On Sunday's Face the Nation, Washington Post columnist Michael Gerson blasted } \\
\text { President Obama for aligning Republicans in Congress with the leadership with Iran } \\
\text { who chant "Death to America" simply for opposing the nuclear deal. }\end{array}$ & 08-09-2015 & NewsBusters \\
\hline & Obama/Liberal & $\begin{array}{l}\text { A comprehensive deal on Iran's nuclear program has been done, diplomats in Vienna } \\
\text { said, bringing to an end a 12-year standoff that had threatened to trigger a new war in } \\
\text { the Middle East. }\end{array}$ & 07-14-2015 & DailyKos \\
\hline \multirow[t]{4}{*}{ Climate Change } & Trump/Liberal & $\begin{array}{l}\text { Much has been made of the fact that Trump's withdrawal from the Paris Climate Agreement } \\
\text { makes the United States an international pariah and a business disaster, that it will undermine } \\
\text { national security, that his excuses were a series of flat-out lies, and that his real reason was the } \\
\text { typical pettiness of his tiny, fragile ego, but the degree to which Trump is Making America } \\
\text { Worst cannot be overstated. }\end{array}$ & 06-02-2017 & DailyKos \\
\hline & Trump/Conservative & $\begin{array}{l}\text { By withdrawing from the agreement, Trump could restore the Senate's constitutional power to } \\
\text { advise on and consent to international treaties. }\end{array}$ & 05-09-2017 & NationalReview \\
\hline & Obama/Conservative & $\begin{array}{l}\text { Wednesday's edition of the CBS Evening News chose to re-air portions of chief medical } \\
\text { correspondent Dr. Jon LaPook's interview with President Obama on climate change supposedly } \\
\text { threatening public health and included LaPook fretting at the end to anchor Scott Pelley that } \\
\text { "climate change legislation has stalled in Congress." }\end{array}$ & 04-08-2015 & NewsBusters \\
\hline & Obama/Liberal & $\begin{array}{l}\text { Because it worked so well to scuttle the global agreement to prevent Iran from getting } \\
\text { a nuclear weapon (not), Republicans are trying the same techniques to undermine the } \\
\text { Paris talks to combat climate change. }\end{array}$ & 09-13-2015 & DailyKos \\
\hline
\end{tabular}

layers are stacked in the concatenation layer. The final layer is fully-connected, then a Softmax activation applied on the final layer output resulting in a class prediction. One drawback from this approach is losing the sequential order of texts, and not being able to model more sophisticated structures.

3) Recurrent Convolutional Neural Networks (RCNN) [61] combines recurrent and convolution layers to take the advantages and mitigate the limitations of both layers. RNN captures contextual semantics by constructing local feature maps of text sentences. Three text representation, left context, right context, and standard word embedding are shared during training update with separate outputs. Forward RNN constructs the left side context, and the right side context is generated by a Reverse RNN. All three text representations are merged by a concatenation layer that fed to a convolution layer. Then a max-pooling layer extracts global most influential feature vectors. The final layer is a fully-connected that passes its output through a Softmax activation function for class prediction.

4) Hierarchical Attention Networks (HAN) [62] best suited for document classification due to its two levels attention mechanisms. Fixed-length of input words encoded by Long-short-term-memory (LSTM) layer. The LSTM itself is wrapped in Bidirectional RNN layer to perform backward RNN computation. The following layer is an attention that forms sentences from the most useful words. Time distributed layer wraps the bidirectional encoder and the attention layer. Another bidirectional RNN layer further encodes the processed sequences to construct a document from the most informative sentences; followed by an attention layer. Finally, a fully-connected layer passes its output to a Softmax activation function to compute the probability of document belonging to a class.

The same networks settings, learning configuration, and text prepossessing techniques depicted in [24] are used in this paper for fair comparison with earlier experiments. Prior to the learning phase, the texts are tokenized based on white space as delimiters. All letter cases are lowered, and punctuation removed from the tokenized texts. The remaining texts are sequenced and padded to 400 sequence length. The highest possible number of features is 35000 unigrams. Word embedding size is 50 with random initialization.

As for the neural network settings, Adaptive Moment Estimation (Adam) is the learning optimizer. The optimizer learning rate is set to 0.0001 and $0.9,0.999$ for the optimizer beta_1 and beta_2 decay parameters, respectively. Both politician penalization and political Ideology detection are single classification problem; therefore, Binary Cross-entropy is selected as a loss function. The model that achieved the best accuracy results on the validation set during training process 


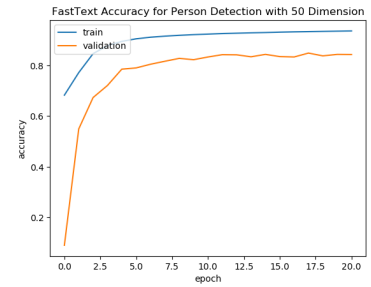

(a) FastText Personalization Detection Accuracy

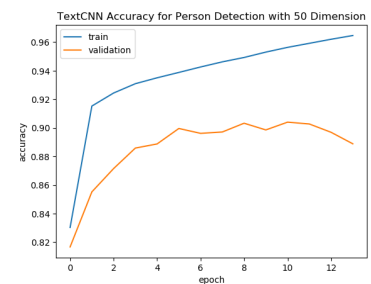

(b) TextCNN Personalization Detection Accuracy

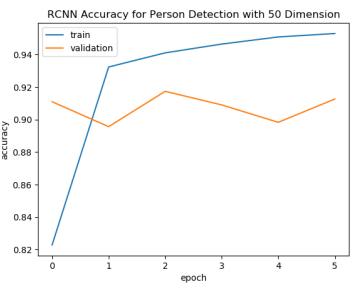

(c) RCNN Personalization Detection Accuracy

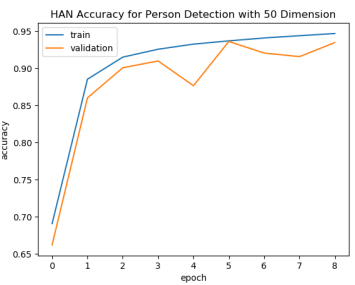

(d) HAN Personalization Detection Accuracy

Fig. 3. Training and Validation Accuracy of Articles' Politicians Personalization Detection Models

is chosen to evaluate the model performance on the testing set. Accuracy as a metric is only used on validation set, other metrics discussed below evaluated models' performance on the testing set. 50 is maximum number of epochs with 32 batch size. Of the training set $15 \%$ is set aside as a validation set to validate model performance posterior to each epoch. With an early-stop scheduler the training will terminate if the validation accuracy did not improve for three consecutive epochs. The networks were built in the Keras platform with TensorFlow as the backend in all the experiments.

During the entire experiment, several metrics evaluated the performance of the neural network models. The best fitted model on the validation set is chosen based on the Accuracy metric, see Equation 1. In binary classification, the accuracy of the model is calculated by dividing the number of correctly predicted examples, which equals to the sum of True Positive (TP) and True Negative, over total number of examples represented by the sum of TP, TN, False Positive (FP), and False Negative (FN).

$$
\text { Accuracy }=\frac{T P+T N}{T P+T N+F P+F N}
$$

While the accuracy metric is a good measure of models performance, it is limited when it comes to imbalanced dataset. Therefore, Precision (Eqn. 2, Recall (Eqn. 3), and F1-score (Eqn. 4) are the measurements metrics used to report the results of detection models on the testing set. Precision computes the fraction of correctly predicted examples of a class among all the examples labeled by the model as the relevant class.

$$
\text { Precision }=\frac{T P}{T P+F P}
$$

On the other hand, Recall derive the fraction of correctly predicted examples of a class among all the examples that actually belong to the relevant class.

$$
\text { Recall }=\frac{T P}{T P+F N}
$$

The harmonic mean of the Precision and Recall or the metric known as F1-score is a metric that overcomes some of the limitations found in other metrics. The F1-score is suitable or imbalanced data and it gives equal importance to Precision and Recall.

$$
F 1=2 * \frac{\text { Precision } * \text { Recall }}{\text { Precision }+ \text { Recall }}=\frac{2 * T P}{2 * T P+F P+F N}
$$

\section{RESUlts AND Discussion}

Constructing ideology detection models trained on personalized set boosts or at least maintain ideology detection performance despite downsizing the training set size. Therefore, this work examines the deep network models' ability to detect articles' personalization. Attaining reasonably satisfactory results in distinguishing among articles centered around particular politician will further prove the existence of political personalization in news media coverage. The deep neural networks trained on articles tagged with politicians, namely Trump and Obama. This paper compares between the results obtained by ideology detection models reported in [24] and personalization detection models. In the subsection $\mathrm{V}-\mathrm{B}$, we test our hypothesis and see if personalized detection models are better at detected the ideology articles than non-personalized ones.

\section{A. Politicians' Personalization Detection Results}

1) Detection Models' Validation Accuracy: It is beneficial to examine the accuracy results of the validation set obtained from the training procedure. The same deep networks employed in the detection of articles' ideology is used, which will assist in observing network behavior trained on the same data but different classes. As depicted in Fig. 3, FastText, Fig. $3 \mathrm{a}$, required 20 epochs before reaching the termination point, which is more than TextCNN 13 epochs, RCNN 5 epochs, and HAN 8 epochs, Fig. 3b, Fig. 3c, and Fig. 3d, respectively.

FastText is the fastest network among the four deep network models. The best accuracy of $93 \%$ on the validation set was achieved by HAN, which is a little bit shy from the training accuracy at its peak. On the other hand, TextCNN accuracy decreased in the last three epochs from $90 \%$ to around $88 \%$. Finally, RCNN was able to recover from descent in epoch 4 to $92 \%$ at the fifth and final epoch. The accuracy results from the validation set do not necessarily reflect the model's performance on the testing set since the models' assessment is done on $15 \%$ of random non-stratified training data.

2) Politicians' Personalization in News Articles: This subsection compares the results of the four deep network models based on three metrics, Precision, Recall, and F1-Score. In Fig. 4, heatmap and bar charts display numerical and graphical 
measurement values for detailed comparison. FastText, Fig. 4a, was the least accurate with $0.92 \mathrm{~F} 1$-Score for Obama, and 0.88 for Trump personalized articles.

All deep network models are better at detecting articles' personalization than ideology. TextCNN and HAN reported the same F1-Score for both classes, Fig. 4b and 4d, but they differ in Recall and Precision. TextCNN is better at detecting Obama articles with 0.95 Recall and 0.92 Precision, yet HAN is less likely to misclassify articles written about Obama as Trump with 0.91 Recall and 0.95 Precision. However, RCNN, Fig. 4c, outperformed other models at detecting Obama with 0.94 Recall, Precision, and F1-Score. As for Trump's articles, all personalization detection networks got $0.91 \mathrm{~F} 1$-Score, 0.93 was the best Recall value recorded by HAN, and 0.93 was the best Precision by TextCNN. In any case, Obama dataset size might be one of the reasons why it received better prediction

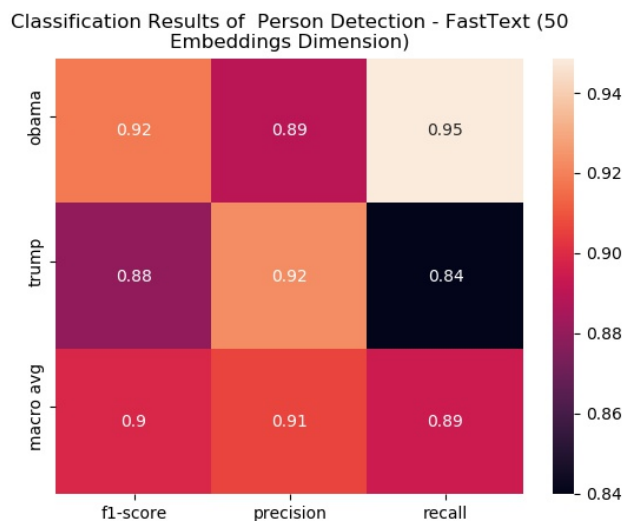

(a) FastText Personalization Detection

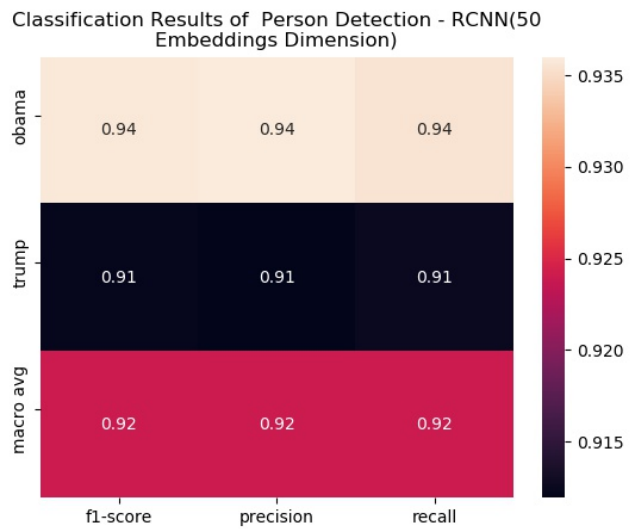

(c) RCNN Personalization Detection

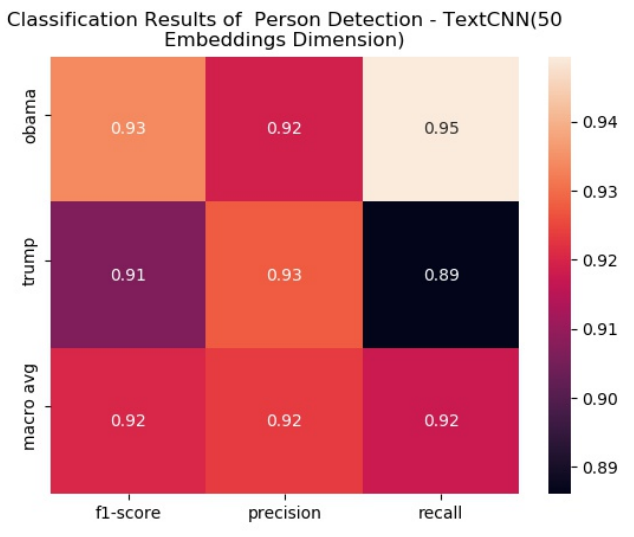

(b) TextCNN Personalization Detection

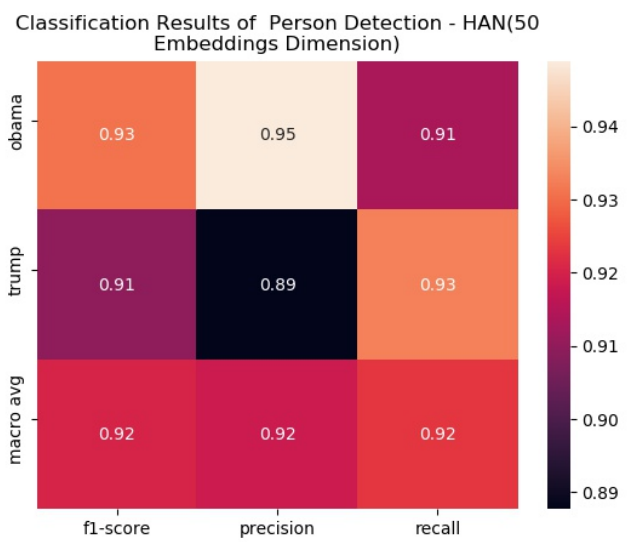

(d) HAN Personalization Detection

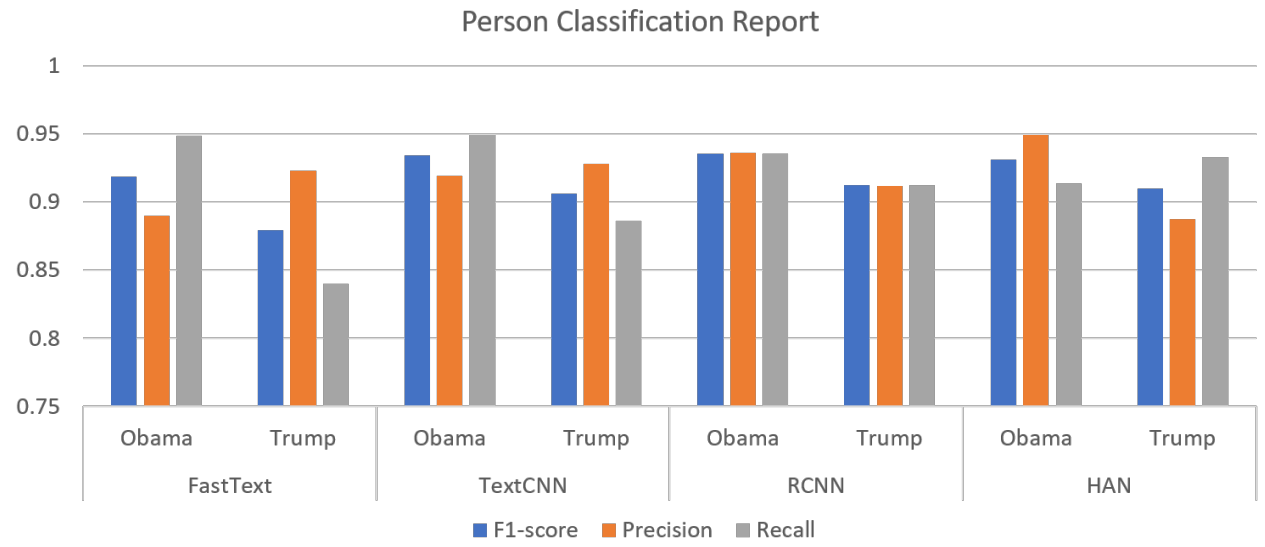

(e) FastText, RCNN, and HAN Personalization Detection Results.

Fig. 4. Personalization Detection Comprehensive Report 


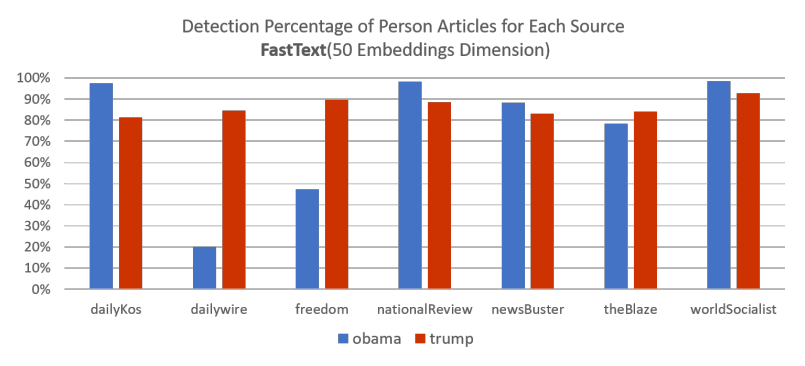

(a) Fasttext Personalization Detection Rate of News Sources

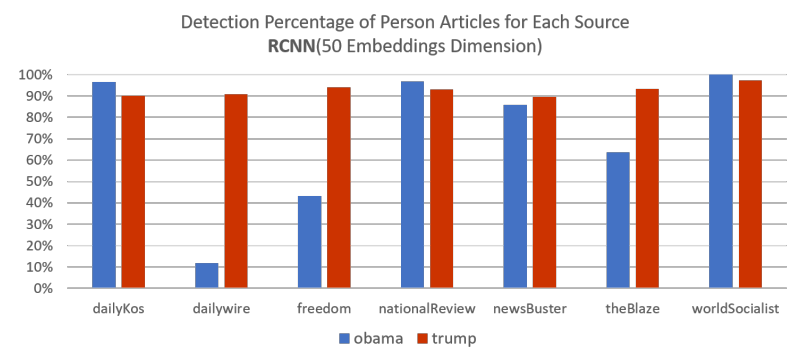

(c) RCNN Personalization Detection Rate of News Sources

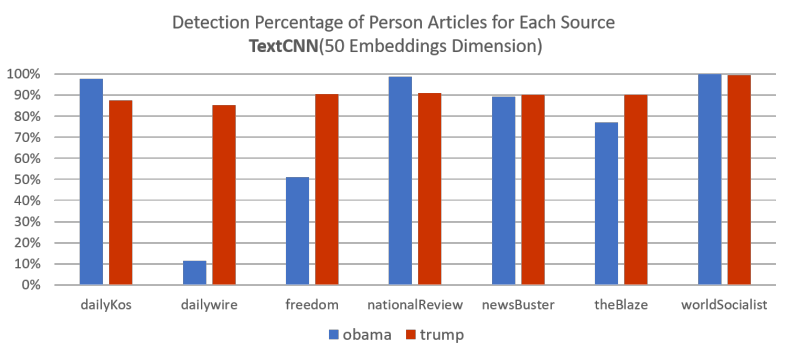

(b) TextCNN Personalization Detection Rate of News Sources

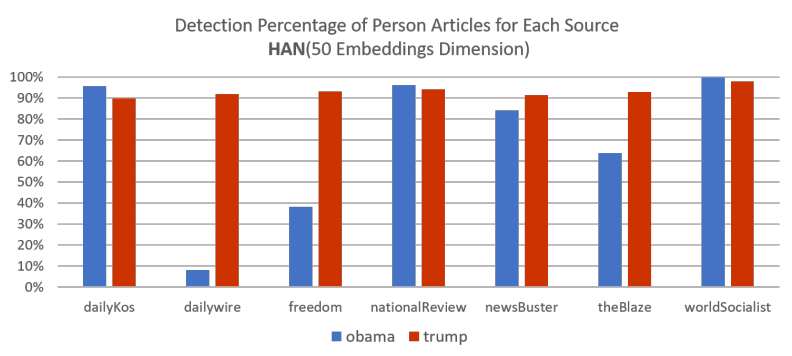

(d) HAN Personalization Detection Rate of News Sources

Fig. 5. Models' Personalization Detection Rate of Presidential Test Articles for All News Sources

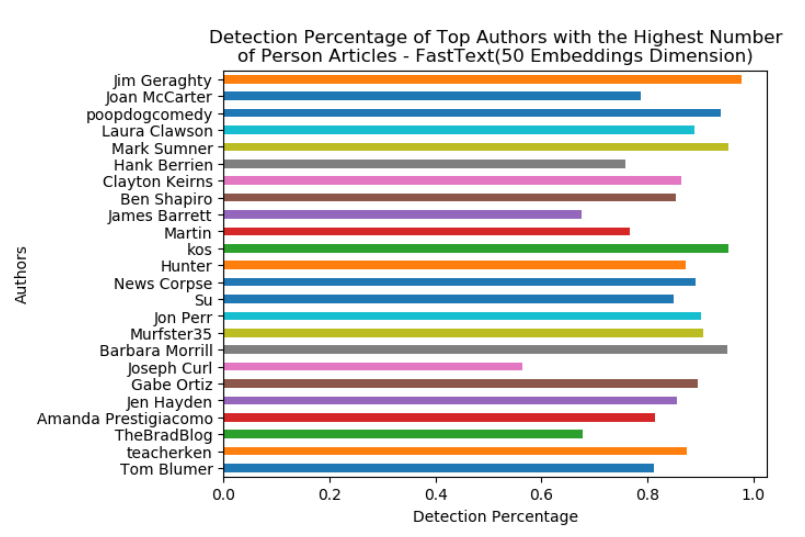

(a) FastText Personalization Detection Rate of Top Authors

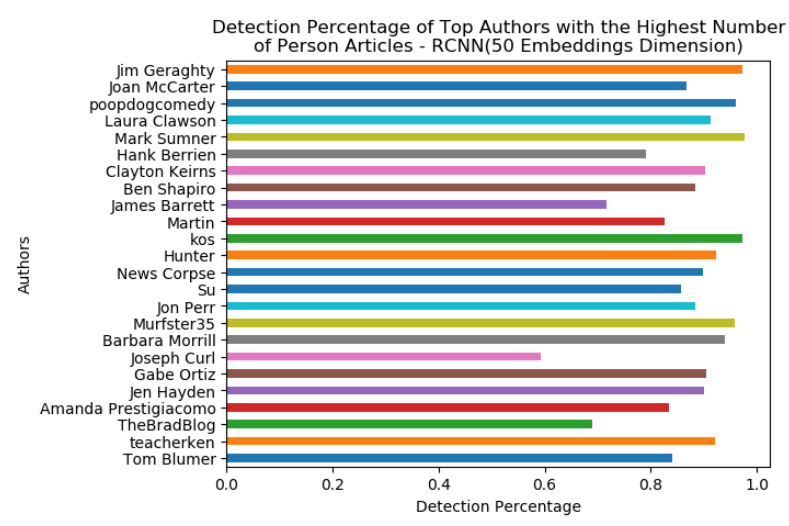

(c) RCNN Personalization Detection Rate of Top Authors

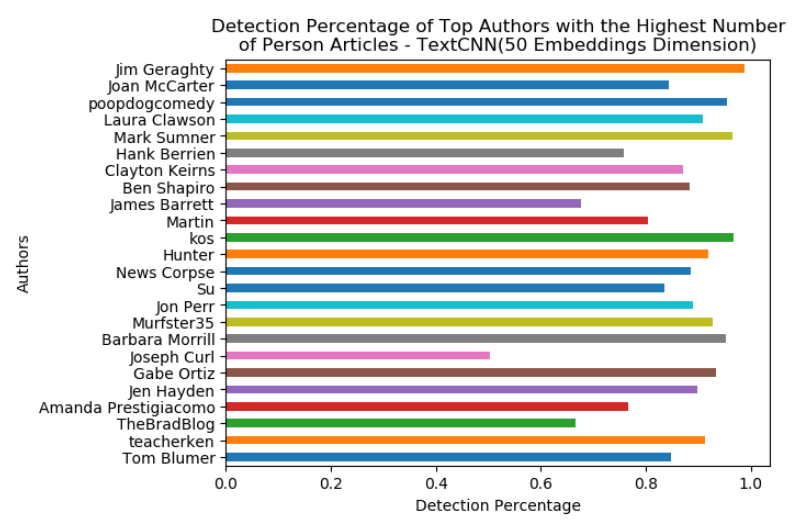

(b) TextCNN Personalization Detection Rate of Top Authors

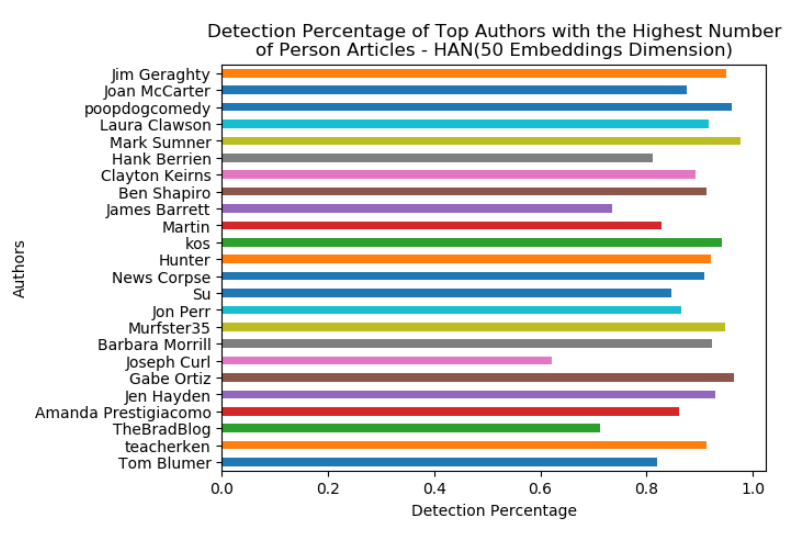

(d) HAN Personalization Detection Rate of Top Authors

Fig. 6. Models' Personalization Detection Percentage for Authors with Highest Number of Published Articles 
results.

3) Politicians' Personalization in Media Sources: Observing news personalization detection percentage based on articles' sources will help identify news sources that maintain politician personalization characteristics across articles, see Fig. 5. It takes us one step further towards news framing and agenda settings detection. News politician personalization is notable in WorldSocialist as one of the far-left news media that might have a particular point of view about Trump and Obama. TextCNN, Fig. 5b, was able to accurately predict $100 \%$ of WorldSocialist's Trump and Obama articles as it has done with detecting articles' ideology. WorldSocialist received high personalization detection accuracy for both classes from the other three models with $100 \%$ for Obama and always above $92 \%$ for Trump. News article personalization does not have to be negative coverage of the politician of interest. Although National Review received non-favorable ideology detection results when labeled as Conservative, it showed that deep neural networks could identify their news articles personalization with more $90 \%$ detection accuracy. DailyKos articles have shown to be more prone to personalizing Obama articles than Trump, though that might be the result of data size skewed towards Obama articles. RCNN, HAN, and FastText (see Fig. 5c, 5d, and 5a) predicted more than $85 \%$ of Trump class articles in the remaining four conservative news websites, namely, DailyWire, IloveMyFreedom, NewsBuster, and TheBlaze, while FastText detected between $82 \%$ and $90 \%$ of Trump articles for the conservative sources. However, Obama's article prediction accuracy originated from the four conservative websites was not as impressive as Trump's articles. HAN detection percentage of politician personalized articles is as low as 9\% of articles personalized on Obama and published by the DailyWire, and $20 \%$ is detected by FastText. It is often believed that data imbalance played a role in this outcome due to having more Trump articles in the three conservative sources than Obama, see Table III. Data imbalance has the same impact on detecting Obama articles originated from IloveMyFreedom, yet to a lesser degree. The gap between Trump and Obama detection accuracy is lower for NewsBusters and the Blaze news media sources. Surprisingly, there are some cases where data imbalance has no significant impact on the personalization detection models outcome, such as the DailyKos and WorldSocialist. This led us to the question of whether the poor prediction accuracy was due to data imbalance.

4) Politicians' Personalization in Authors: Authors personalize articles by shaping a persona that fits his or her point of view about the targeted politician. Unlike article ideologies, where authors compose articles that follow an ideology, news personalization is harder to define. Politically motivated authors will not easily alter their perception; hence, articles will consistently follow a pattern that might be identified. Some of the authors who had low ideology detection accuracy, got high detection accuracy for personalized articles, such as Jim Geraghty, see Fig. 6. This could mean the author has a fixated opinion about the politician in interest, but his point of view does not align with a specific ideology. The opposite is also possible where the author's articles' ideology is identified more accurately than its personalization as for TheBradBlog. Moreover, the third possible scenario where the prediction accuracy of articles' ideology and personalization of an author are both high as in Pooddogcomedy, Markos Moulitsas, and KOS. The author with the least personalized detection accuracy is Joseph Curl, with approximately $60 \%$ detection rate.

\section{B. Detecting Articles' Political Ideology with Personalized- Based Models}

Unlike conventional linear text classifiers such SVM, deep networks require a large sum of data to deliver on various tasks, including text detection. However, learning the model on personalized articles improves or maintains ideology detection models' performance, even though the personalized training sets are a subset of the Presidential training set.

The previous statement validity is verified by training the deep neural networks on the Presidential, Trump, and Obama articles separately. Then, test the ideology detection models on Presidential, Trump, and Obama testing sets. The results obtained by those experiments are compared to each other to identify which approach is more suitable for this problem.

Fig. 7 and Fig. 8 illustrate the results of deep networks trained on the Presidential, Trump, and Obama training sets, and tested on the personalized sets only. Logically, one should expect that the accuracy of the ideology detection models will decrease when trained on a subset of the entire training set since the model will lose some information by removing a large chunk of training data. However, training on Trump set alone, Fig. 7, to detect the ideology of articles written about President Donald Trump resulted in better performance, despite removing $58 \%$ of the training set. All four deep network models scored higher on Precision, Recall, and F1Score for Conservative and Liberal classes. Except for RCNN, Fig. 7c, that reported lower recall for the Liberal class when trained on Trump data, other metrics have increased. TextCNN, Fig. 7b, still the best ideology detection model with training on personalized data f1-score improved from 0.873 to 0.899 for the Conservative class and 0.904 to 0.924 for the Liberal class.

The experiment is extended to test out the ideology detection model performance when trained on Obama set to predict the ideology of articles in the Trump testing set. Although the Obama dataset is more significant in size than Trump, ideology models performed poorly compared to detection models trained on Trump or the Presidential training sets. Despite the fact these articles were collected from the same sources, the performance of models widely differs, which proved that news personalization has an impact on news article ideology detection.

Furthermore, all four deep networks retrained on Obama training set alone to predict the ideology of Obama's articles. Fig. 8a shows that FastText F1-Score slightly improved from 0.789 to 0.804 for Conservative and 0.960 to 0963 for Liberal. Other network models did not show any improvement, yet no severe decrease in their performance either. Relatively, the size of the data removed from the Presidential training set is still significant compared to information loss measure by detection model performance. Removing $42 \%$ from the entire training set did not have a severe impact on Obama's article ideology detection models. The model's performance drastically degraded when trained on Trump training set alone. The articles were collected from multiple sources with diverse topics and share 


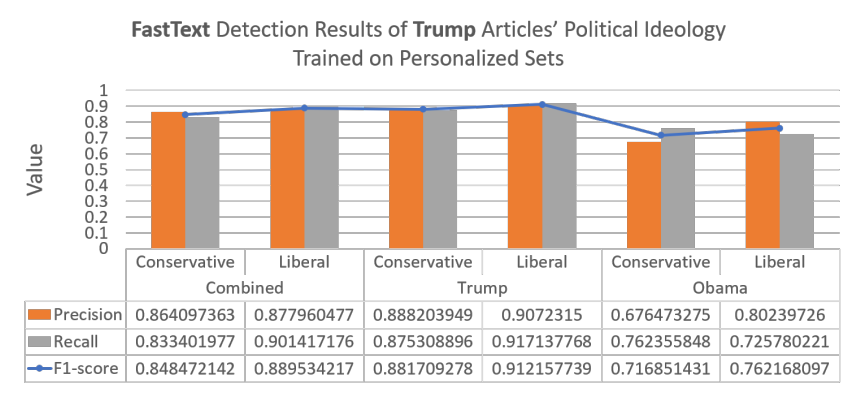

(a) FastText Ideology Detection Results of Trump Test Set

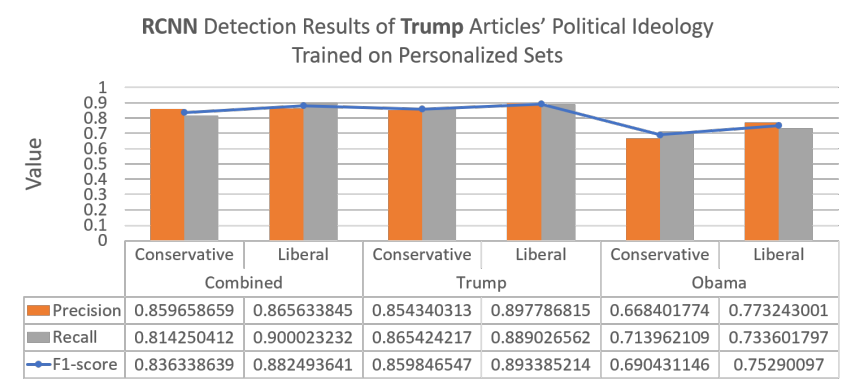

(c) RCNN Ideology Detection Results of Trump Test Set

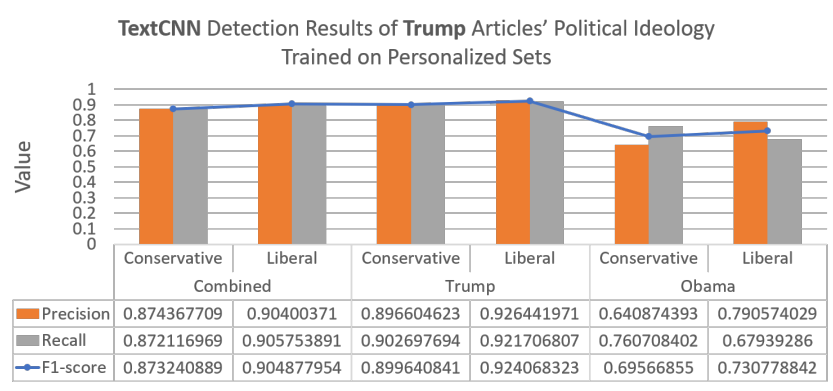

(b) TextCNN Ideology Detection Results of Trump Test Set

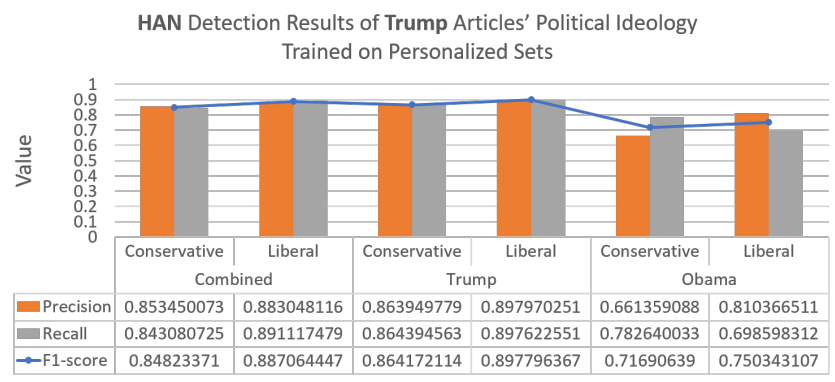

(d) HAN Ideology Detection Results of Trump Test Set

Fig. 7. Detection Results of Network Models Learned on Presidential and Personalized Training Sets to Predict the Ideology of Trump Test Articles.

a person of interest. Therefore, we believe that the results obtained from training models on the personalized sets are evidence that political personalization exists in news media articles. Reconstructing data based on personalization provides coherency and logical relation among the data, making it easier for deep networks to identify different ideological traits.

\section{CONCLUSION}

We successfully implemented neural networks models that accurately detected politicians' personalization and political

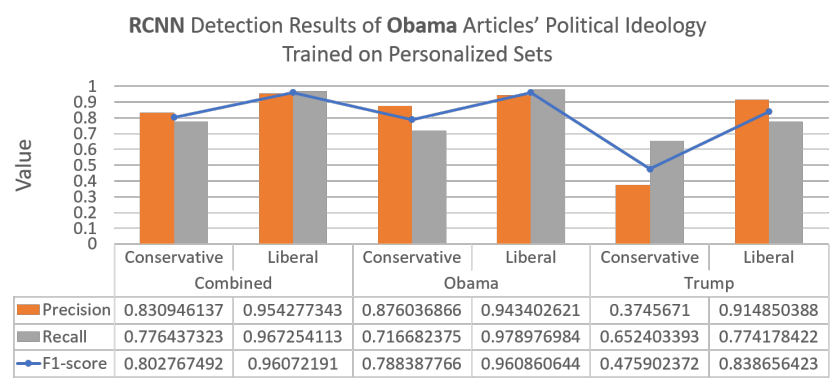

(b) TextCNN Ideology Detection Results of Obama Test Set

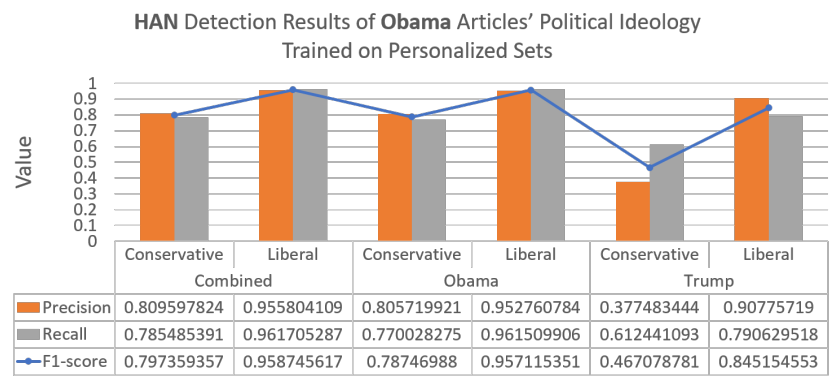

(d) HAN Ideology Detection Results of Obama Test Set

(c) RCNN Ideology Detection Results of Obama Test Set

Fig. 8. Detection Results of Network Models Learned on Presidential and Personalized Training Sets to Predict the Ideology of Obama Test Articles. 
ideology in news articles, authors, and media sources. This work proved that some authors are consistent in their politicians' coverage style and more politically affiliated. Although with different degrees of bias, media sources exhibited patterns in selecting published articles. However, detecting politicians' personalization in news media is a new research topic that needs further examination. We are not aware of any research papers that studied the relation between more definitive influencing mechanisms and politicians' personalization, which will lead to new research directions that combine political science and artificial intelligence. One way to improve the work in this paper is by expanding the dataset to include more politicians or political ideologies and revaluating the detection models' performance on multiclass problems. Also, end-to-end deep neural networks can solve hierarchal problems to identify politicians' personalization and political ideology with a single network. Furthermore, other deep neural networks, such as BERT, and pre-trained networks, might achieve better results on the Presidential dataset.

\section{ACKNOWLEDGMENT}

The authors would like to thank the Deanship of Scientific Research at Umm Al-Qura University for supporting this work by Grant Code: (22UQU4340018DSR01).

\section{REFERENCES}

[1] J. Dunaway, K. Searles, M. Sui, and N. Paul, "News attention in a mobile era," Journal of Computer-Mediated Communication, vol. 23, no. 2, pp. 107-124, 2018.

[2] S. Schäfer, "Illusion of knowledge through facebook news? effects of snack news in a news feed on perceived knowledge, attitude strength, and willingness for discussions," Computers in Human Behavior, vol. 103, pp. 1-12, 2020

[3] H. Wen, L. Fang, and L. Guan, "A hybrid approach for personalized recommendation of news on the web," Expert Systems with Applications, vol. 39, no. 5, pp. 5806-5814, 2012.

[4] H. J. Lee and S. J. Park, "Moners: A news recommender for the mobile web," Expert Systems with Applications, vol. 32, no. 1, pp. 143-150, 2007.

[5] R. C. Bagher, H. Hassanpour, and H. Mashayekhi, "User trends modeling for a content-based recommender system," Expert Systems with Applications, vol. 87, pp. 209-219, 2017.

[6] A. Montes-García, J. M. Álvarez-Rodríguez, J. E. Labra-Gayo, and M. Martínez-Merino, "Towards a journalist-based news recommendation system: The wesomender approach," Expert Systems with Applications, vol. 40, no. 17, pp. 6735-6741, 2013.

[7] K. Thorson, K. Cotter, M. Medeiros, and C. Pak, "Algorithmic inference, political interest, and exposure to news and politics on facebook," Information, Communication \& Society, pp. 1-18, 2019.

[8] G. de Souza Pereira Moreira, F. Ferreira, and A. M. da Cunha, "News session-based recommendations using deep neural networks," in Proceedings of the 3rd Workshop on Deep Learning for Recommender Systems, 2018, pp. 15-23.

[9] V. Kumar, D. Khattar, S. Gupta, M. Gupta, and V. Varma, "Deep neural architecture for news recommendation." in CLEF (Working Notes), 2017.

[10] C. Chen, X. Meng, Z. Xu, and T. Lukasiewicz, "Location-aware personalized news recommendation with deep semantic analysis," IEEE Access, vol. 5, pp. 1624-1638, 2017.

[11] I. Dylko, I. Dolgov, W. Hoffman, N. Eckhart, M. Molina, and O. Aaziz, "The dark side of technology: An experimental investigation of the influence of customizability technology on online political selective exposure," Computers in Human Behavior, vol. 73, pp. 181-190, 2017.
[12] N. M. Anspach, "The new personal influence: How our facebook friends influence the news we read," Political Communication, vol. 34, no. 4, pp. 590-606, 2017.

[13] K. Sasahara, W. Chen, H. Peng, G. L. Ciampaglia, A. Flammini, and F. Menczer, "Social influence and unfollowing accelerate the emergence of echo chambers," Journal of Computational Social Science, pp. 1-22, 2020.

[14] M. E. Peters, M. Neumann, M. Iyyer, M. Gardner, C. Clark, K. Lee, and L. Zettlemoyer, "Deep contextualized word representations," arXiv preprint arXiv:1802.05365, 2018.

[15] C. Qu, F. Ji, M. Qiu, L. Yang, Z. Min, H. Chen, J. Huang, and W. B. Croft, "Learning to selectively transfer: Reinforced transfer learning for deep text matching," in Proceedings of the Twelfth ACM International Conference on Web Search and Data Mining, 2019, pp. 699-707.

[16] P. Liu, X. Qiu, and X. Huang, "Adversarial multi-task learning for text classification," arXiv preprint arXiv:1704.05742, 2017.

[17] J. T. Hamilton and R. Zeckhauser, "Media coverage of ceos: who? what? where? when? why?" Unpublished working paper, Stanford Institute of International Studies, 2004.

[18] F. Bendisch, G. Larsen, and M. Trueman, "Fame and fortune: a conceptual model of ceo brands," European Journal of Marketing, 2013.

[19] Y. Mehta, N. Majumder, A. Gelbukh, and E. Cambria, "Recent trends in deep learning based personality detection," Artificial Intelligence Review, pp. 1-27, 2019.

[20] O. Tsur, D. Calacci, and D. Lazer, "A frame of mind: Using statistical models for detection of framing and agenda setting campaigns," in Proceedings of the 53rd Annual Meeting of the Association for Computational Linguistics and the 7th International Joint Conference on Natural Language Processing (Volume 1: Long Papers), 2015, pp. 1629-1638.

[21] Z. Ahmad, R. Jindal, A. Ekbal, and P. Bhattachharyya, "Borrow from rich cousin: transfer learning for emotion detection using cross lingual embedding," Expert Systems with Applications, vol. 139, p. 112851, 2020.

[22] F. Enríquez, J. A. Troyano, and T. López-Solaz, "An approach to the use of word embeddings in an opinion classification task," Expert Systems with Applications, vol. 66, pp. 1-6, 2016.

[23] F. Hamborg, K. Donnay, and B. Gipp, "Automated identification of media bias in news articles: an interdisciplinary literature review," International Journal on Digital Libraries, vol. 20, no. 4, pp. 391-415, 2019.

[24] K. M. Alzhrani, "Political ideology detection of news articles using deep neural networks," Intelligent Automation \& Soft Computing, vol. 33, no. 1, pp. 483-500, 2022.

[25] D. Ball, P. S. Coelho, and M. J. Vilares, "Service personalization and loyalty," Journal of services marketing, 2006.

[26] W. Meng, R. Ding, S. P. Chung, S. Han, and W. Lee, "The price of free: Privacy leakage in personalized mobile in-apps ads." in NDSS, 2016.

[27] F. Essalmi, L. J. B. Ayed, M. Jemni, S. Graf et al., "A fully personalization strategy of e-learning scenarios," Computers in Human Behavior, vol. 26, no. 4, pp. 581-591, 2010.

[28] O. Ben-Shahar and A. Porat, "Personalizing negligence law," NYUL Rev., vol. 91, p. 627, 2016.

[29] J. Vesanen, "What is personalization? a conceptual framework," European Journal of Marketing, 2007.

[30] H. Fan and M. S. Poole, "What is personalization? perspectives on the design and implementation of personalization in information systems," Journal of Organizational Computing and Electronic Commerce, vol. 16, no. 3-4, pp. 179-202, 2006.

[31] B. Maddens and S. Fiers, "The direct pm election and the institutional presidentialisation of parliamentary systems," Electoral Studies, vol. 23, no. 4, pp. 769-793, 2004.

[32] D. R. Kinder, M. D. Peters, R. P. Abelson, and S. T. Fiske, "Presidential prototypes," Political behavior, vol. 2, no. 4, pp. 315-337, 1980.

[33] F. F. da Silva, D. Garzia, and A. De Angelis, "From party to leader mobilization? the personalization of voter turnout," Party Politics, 2019.

[34] T. Bøggild and H. H. Pedersen, "Campaigning on behalf of the party? party constraints on candidate campaign personalisation," European Journal of Political Research, vol. 57, no. 4, pp. 883-899, 2018. 
[35] S. Kruikemeier, G. Van Noort, R. Vliegenthart, and C. H. De Vreese, "Getting closer: The effects of personalized and interactive online political communication," European journal of communication, vol. 28, no. 1, pp. 53-66, 2013.

[36] I. McAllister et al., "The personalization of politics," in The Oxford handbook of political behavior. Oxford University Press, 2007.

[37] S. Mainwaring and M. Torcal, "Party system institutionalization and party system theory after the third wave of democratization," Handbook of party politics, vol. 11, no. 6, pp. 204-227, 2006.

[38] A. Mughan, Media and the presidentialization of parliamentary elections. Springer, 2000.

[39] T. Poguntke and P. Webb, The presidentialization of politics: A comparative study of modern democracies. Oxford University Press on Demand, 2007.

[40] _ "The presidentialization of politics in democratic societies: A framework for analysis," The presidentialization of politics: a comparative study of modern democracies, vol. 1, 2005.

[41] P. Webb and T. Poguntke, "The presidentialisation of politics thesis defended," Parliamentary Affairs, vol. 66, no. 3, pp. 646-654, 2013.

[42] K. Dowding, "The prime ministerialisation of the british prime minister," Parliamentary Affairs, vol. 66, no. 3, pp. 617-635, 2013.

[43] D. Garzia, Personalization of politics and electoral change. Springer, 2019.

[44] H. Kriesi, "The populist challenge," West European Politics, vol. 37, no. 2, pp. 361-378, 2014.

[45] M. Canovan, "Trust the people! populism and the two faces of democracy," Political studies, vol. 47, no. 1, pp. 2-16, 1999.

[46] A. I. Langer and I. Sagarzazu, "Bring back the party: personalisation, the media and coalition politics," West European Politics, vol. 41, no. 2, pp. 472-495, 2018.

[47] A. Bittner, Platform or personality?: the role of party leaders in elections. OUP Oxford, 2011.

[48] L. Karvonen, The personalisation of politics: A study of parliamentary democracies. Ecpr Press, 2010.

[49] M. Kaase, "Is there personalization in politics? candidates and voting behavior in Germany," International Political Science Review, vol. 15, no. 3, pp. 211-230, 1994.

[50] P. Van Aelst, T. Sheafer, and J. Stanyer, "The personalization of medi- ated political communication: A review of concepts, operationalizations and key findings," Journalism, vol. 13, no. 2, pp. 203-220, 2012.

[51] M. Balmas, G. Rahat, T. Sheafer, and S. R. Shenhav, "Two routes to personalized politics: Centralized and decentralized personalization," Party Politics, vol. 20, no. 1, pp. 37-51, 2014.

[52] M. Balmas and T. Sheafer, "Leaders first, countries after: Mediated political personalization in the international arena," Journal of соттиnication, vol. 63, no. 3, pp. 454-475, 2013.

[53] J. Takens, J. Kleinnijenhuis, A. Van Hoof, and W. Van Atteveldt, "Party leaders in the media and voting behavior: Priming rather than learning or projection," Political Communication, vol. 32, no. 2, pp. 249-267, 2015.

[54] B. Wauters, P. Thijssen, P. Van Aelst, and J.-B. Pilet, "Centralized personalization at the expense of decentralized personalization. the decline of preferential voting in belgium (2003-2014)," Party Politics, vol. 24, no. 5, pp. 511-523, 2018.

[55] G. Rahat and T. Sheafer, "The personalization (s) of politics: Israel, 1949-2003," Political communication, vol. 24, no. 1, pp. 65-80, 2007.

[56] S. Adam and M. Maier, "Personalization of politics a critical review and agenda for research," Annals of the International Communication Association, vol. 34, no. 1, pp. 213-257, 2010.

[57] K. Alzhrani, "Ideology detection of personalized political news coverage: A new dataset," in Proceedings of the 2020 the 4th International Conference on Compute and Data Analysis, 2020, pp. 10-15.

[58] Y. Li, G. Sun, and Y. Zhu, "Data imbalance problem in text classification," in 2010 Third International Symposium on Information Processing. IEEE, 2010, pp. 301-305.

[59] A. Joulin, E. Grave, P. Bojanowski, and T. Mikolov, "Bag of tricks for efficient text classification," arXiv preprint arXiv:1607.01759, 2016.

[60] Y. Kim, "Convolutional neural networks for sentence classification," arXiv preprint arXiv:1408.5882, 2014.

[61] S. Lai, L. Xu, K. Liu, and J. Zhao, "Recurrent convolutional neural networks for text classification," in Twenty-ninth AAAI conference on artificial intelligence, 2015.

[62] Z. Yang, D. Yang, C. Dyer, X. He, A. Smola, and E. Hovy, "Hierarchical attention networks for document classification," in Proceedings of the 2016 conference of the North American chapter of the association for computational linguistics: human language technologies, 2016, pp. 1480-1489. 